\title{
Is intracellular pH a clock for mitosis?
}

\author{
L John Gagliardi ${ }^{1}$ and Daniel H Shain ${ }^{2 *}$
}

\footnotetext{
* Correspondence: dshain@camden. rutgers.edu

${ }^{2}$ Department of Biology, Rutgers The State University of New Jersey, Camden, NJ 08102, USA

Full list of author information is available at the end of the article
}

\begin{abstract}
Experiments have shown that the intracellular $\mathrm{pH}$ of many cells rises to a maximum at the onset of mitosis, subsequently decreasing 0.3 to $0.5 \mathrm{pH}$ units by the end of mitosis. This result, and observations that tubulin net charge depends strongly on $\mathrm{pH}$, may be critical for microtubule (MT) dynamics during mitosis. In vivo studies demonstrate that MT dynamics is sensitive to $\mathrm{pH}$, with MT growth favored by higher $\mathrm{pH}$ values. Therefore it seems likely that the shift from the dominance of microtubule growth during prophase, and to a lesser extent during prometaphase, to a parity between MT polymerization and depolymerization during metaphase chromosome oscillations is a consequence of gradually decreasing intracellular $\mathrm{pH}$ during mitosis. Thus the timing and sequencing of prophase, prometaphase, and metaphase chromosome motions may be understood as an increase in the MT disassembly to assembly probability ratio resulting from a continuously declining intracellular $\mathrm{pH}$.
\end{abstract}

\section{Introduction}

In the cytoplasmic medium (cytosol) within biological cells, it is generally thought that electrostatic fields are subject to strong attenuation by screening with oppositely charged ions (counterion screening), decreasing exponentially to much smaller values over a distance of several Debye lengths. The Debye length (distance over which the electric field decreases to approximately $36.8 \%$ of the previous value) within cells is typically given as $1 \mathrm{~nm}$ [1], and since eukaryotic cells have much larger dimensions one is tempted to conclude that electrostatics is not a major factor in explaining mitotic chromosome movements. However, the presence of microtubules, as well as other factors discussed below, force this assumption to be reconsidered.

The characteristics of microtubule lengthening (polymerization) and shortening (depolymerization) follow a pattern known as "dynamic instability": that is, at any given instant some microtubules are growing while others are undergoing rapid breakdown. In general, the rate at which microtubules undergo net assembly - or disassembly - varies with mitotic stage [2]. Changes in microtubule dynamics are integral to changes in chromosome motions during mitotic stages. Poleward and antipoleward chromosome motions occur during prometaphase and metaphase. Antipoleward motions dominate during the congressional movement of chromosomes to the cell equator, and poleward motion prevails during anaphase A. It is assumed here that poleward chromosome motions are in response to disassembling kinetochore microtubules at kinetochores and poles, and argued elsewhere [3] that antipoleward chromosome motions are best explained by assembling microtubules at chromosome arms.

(c) 2013 Gagliardi and Shain; licensee BioMed Central Ltd. This is an Open Access article distributed under the terms of the Creative Commons Attribution License (http://creativecommons.org/licenses/by/2.0), which permits unrestricted use, distribution, and reproduction in any medium, provided the original work is properly cited. 
Experiments have shown that intracellular $\mathrm{pH}\left(\mathrm{pH}_{\mathrm{i}}\right)$ of many cells rises to a maximum at the onset of mitosis, subsequently falling steadily through cell division. Although it is experimentally difficult to resolve the starting time for the beginning of $\mathrm{pH}_{\mathrm{i}}$ decrease during the cell cycle, it appears to drop 0.3 to $0.5 \mathrm{pH}$ units from typical peak values of 7.3 to 7.5 measured during prophase when microtubule polymerization is favored $[4,5]$.

Studies have shown that in vivo microtubule polymerization is favored by higher $\mathrm{pH}$ values [6], in contrast with in vitro studies which suggest a $\mathrm{pH}$ optimum in the range of 6.3 to 6.9. The disagreement between these values has been considered in relation to the nucleation potential of microtubule organizing centers like centrosomes [6], suggesting that $\mathrm{pH}_{\mathrm{i}}$ regulates the nucleation potential of microtubule organizing centers $[7,8]$. Experiments have also shown that ionic concentrations play an important role in microtubule polymerization [9]. Taken together, these observations seem to favor the more complex physiology of in vivo analyses to resolve this question.

Cellular electrostatics is strongly influenced by reduced counterion screening due to layered water adhering to charged molecules. Such water layering - with consequent reduction or elimination of Debye screening - at charged proteins has long been theorized $[10,11]$ and has been confirmed experimentally [12]. Additionally, water between sufficiently close (up to $3 \mathrm{~nm}$ ) charged proteins has a dielectric permittivity that is considerably reduced from the bulk value far from charged surfaces [13-15]. The combination of these two effects (or conditions) - water layering and reduced dielectric constant - can influence cellular electrostatics in a number of important ways. This is especially true in relation to mitosis [15]. For example, these conditions further increase the tendency for an electrostatic assist to aster and spindle self-assembly (see below).

A number of investigations have focused on the electrostatic properties of microtubule tubulin subunits [16-19]. Large scale calculations of tubulin have been conducted using molecular dynamics programs along with protein parameter sets [20]. The dipole moment of tubulin has been calculated to be as large as 1,800 Debye units (D) [17,21]. In experiments conducted near physiological conditions, the dipole moment was $36 \mathrm{D}$ [22], corresponding to a dipole charge of approximately 0.1 electron per dimer. Experiments have shown that tubulin net charge depends strongly on $\mathrm{pH}$, varying quite linearly from -12 to -28 (electron charges) between $\mathrm{pH} 5.5$ and 8.0 [21,23]. This could be important for microtubule dynamics during mitosis because, as noted above, many cell types exhibit a decrease of 0.3 to $0.5 \mathrm{pH}$ units during mitosis.

Tubulin has a large overall negative charge of 20 at $\mathrm{pH} 7$ and as much as $40 \%$ of the charge resides on the $\mathrm{C}$-termini, which extend outward from the microtubule axis as a function of $\mathrm{pH}_{\mathrm{i}}$ (e.g., 4-5 nm at $\mathrm{pH}_{\mathrm{i}} 7$ [20]). It seems likely therefore that an increased tubulin charge and the resulting greater extension of $\mathrm{C}$-termini may be integral to an increased probability for microtubule assembly during prophase when $\mathrm{pH}_{\mathrm{i}}$ is highest [5].

\section{Intracellular pH as a clock for mitosis}

In addition to addressing force generation for post-attachment chromosome motions $[3,15,24]$, a continuum electrostatics approach to mitotic motions can also account for the timing and sequencing of the detailed changes in these motions. These can be attributed to changes in microtubule dynamics based on a progressively increasing microtubule 
disassembly to assembly ratio for kinetochore microtubules that is caused by a steadily decreasing $\mathrm{pH}_{\mathrm{i}}$ during mitosis.

It therefore seems reasonable to expect that prophase high $\mathrm{pH}_{\mathrm{i}}$ conditions and the electrostatic nature of tubulin dimer subunits greatly assists in their self-assembly into the microtubules of the asters and spindle. As pHi increases beyond interphase the presence of nucleating centers, along with the favoring of microtubule polymerization in a higher pHi environment, suggests that the pool of tubulin from interphase microtubule disassembly will polymerize around prophase centrosomes. As discussed in the previous section, this self-assembly would be aided by reduced counterion screening due to layered water and the reduced dielectric constant between charged protein surfaces. An electrostatic component to the biochemistry of the microtubules in assembling asters is consistent with experimental observations of $\mathrm{pH}$ effects on microtubule assembly [6], as well as the sensitivity of microtubule stability to calcium ion concentrations [25,26].

The two effects (or conditions) discussed above are expected to increase the efficiency of microtubule self -assembly in asters and spindles by (1) allowing electrostatic interactions over greater distances than Debye (counterion) screening dictates, and (2) further increasing the strength of these interactions by an order of magnitude due to a corresponding order of magnitude reduction in the cytosolic dielectric constant between charged protein surfaces separated by critical distances.

Thus it seems reasonable to assume that, over distances consistent with the reduced dielectric constant and modified counterion screening, the electrostatic nature of tubulin dimers allows tubulin dimer microtubule subunits to align end-to-end and laterally, facilitating the formation of asters and mitotic spindles [24,27].

Similarly, a mutually repulsive electrostatic force between subsets of like-charged free plus ends of interacting microtubules from opposite half-spindles in the growing mitotic spindle is expected to increase in magnitude and range. Thus mutual electrostatic repulsion of negatively charged microtubule plus ends distal to centrosomes in assembling asters/half-spindles could provide the driving force for their poleward migration in the forming spindle [15,27]. A subset of interacting microtubules in a small portion of a forming spindle is depicted in Figure 1.

Interacting microtubules can result from either growing or shrinking microtubules but polymerization probabilities will dominate during prophase. An increased probability for microtubule depolymerization, as compared to the prophase predominance of microtubule assembly, is consistent with experimental observations of alternating poleward and antipoleward motions - with antipoleward motions more probable - of monovalently attached chromosomes during prometaphase. As discussed elsewhere [3,24], after a bivalent attachment to both poles, electrostatic poleward forces toward both poles acting in conjunction with inverse square antipoleward forces exerted between negatively charged microtubule free plus ends and negatively charged chromosome arms could account for chromosome congression. Metaphase chromosome mid-cell oscillations are indirect experimental evidence for a microtubule disassembly to assembly (disassembly/ assembly) probability ratio approaching unity as $\mathrm{pH}_{\mathrm{i}}$ continues to decline.

At late metaphase, before anaphase-A, experiments reveal that the poleward motions of sister kinetochores stretch the intervening centromeric chromatin, producing high kinetochore tensions [28]. These high tensions are likely attributed to a continuing microtubule disassembly/assembly probability ratio increase caused by a continuously 


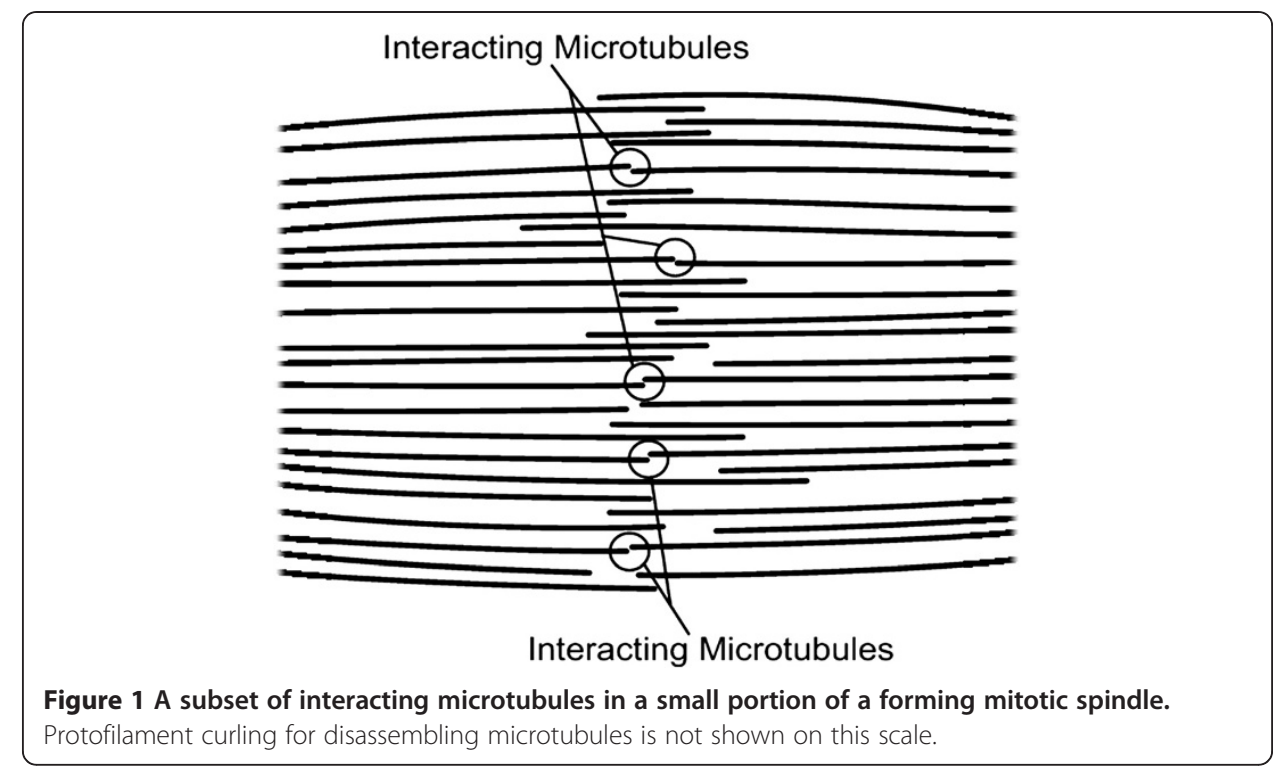

lowering $\mathrm{pH}_{\mathrm{i}}$. The resulting attendant increase in poleward disassembly force on sister chromatids would lead to increased tension.

Regarding post-attachment chromosome motions through metaphase, it seems reasonable to ascribe an increasing dissassembly/assembly probability ratio - with attendant changes in microtubule dynamics and associated mitotic chromosome motions through metaphase - to an experimentally-observed steadily decreasing $\mathrm{pH}_{\mathrm{i}}$. We may then envision a decrease in $\mathrm{pH}_{\mathrm{i}}$ from a peak at prophase favoring microtubule assembly, declining through prometaphase and continuing to decline through metaphase when parity between microtubule assembly and disassembly leads to mid-cell chromatid pair oscillation, culminating in increased microtubule disassembly-associated kinetochore tension late in metaphase, as the cell's master clock controlling microtubule dynamics, and consequently the events of mitosis. One might also be tempted to attribute the more complete dominance of microtubule disassembly - with an accompanying predominance of poleward disassembly forces at kinetochores and poles - during anaphase-A to a further continuation of decreasing intracellular $\mathrm{pH}$. However, as discussed elsewhere [3,15], any additional lowering of $\mathrm{pH}_{\mathrm{i}}$ after metaphase may work in conjunction with increased $\left[\mathrm{Ca}^{2+}\right]$ $[29,30]$ as major determinants of anaphase- $\mathrm{A}$ and anaphase-B motions.

\section{Conclusions}

High $\mathrm{pH}_{\mathrm{i}}$ during prophase favors spindle assembly. This includes greater electrostatic attractive forces between tubulin dimers as well as increased repulsive electrostatic interactions between growing microtubule plus ends driving poleward movement of forming half-spindles. Due to reduced counterion screening and the low dielectric constant of layered water adhering to charged tubulin dimers, the necessary attraction and alignment of tubulin dimers during spindle self-assembly would be enhanced by the considerably increased range and strength of electrostatic attractions between oppositely charged regions. 
Changes in microtubule dynamics are integral to changes in chromosome motions during mitosis, and can be attributed to an associated change in intracellular $\mathrm{pH}\left(\mathrm{pH}_{\mathrm{i}}\right)$. In particular, a decrease in $\mathrm{pH}_{\mathrm{i}}$ through mitosis may act as a master clock controlling microtubule disassembly/assembly probability ratios by altering the electrostatic interactions of tubulin dimers. This, in turn, would determine the timing and dynamics of post-attachment mitotic chromosome motions through metaphase.

Thus it seems reasonable to assume that the shift from the dominance of microtubule growth during prophase, to a lesser extent during prometaphase, and to approximate parity between microtubule polymerization and depolymerization during metaphase chromosome oscillations, can be attributed to the gradual downward $\mathrm{pH}_{\mathrm{i}}$ shift during mitosis that is observed in many eukaryotic cells.

Competing interests

The authors declare that they have no competing interests.

Authors' contributions

LJG conceptualized the theoretical aspects of this article and DHS provided intellectual contributions. Both authors read and approved the final manuscript.

\section{Author details}

${ }^{1}$ Department of Physics, Rutgers The State University of New Jersey, Camden, NJ 08102, USA. ²Department of Biology, Rutgers The State University of New Jersey, Camden, NJ 08102, USA.

Received: 13 January 2013 Accepted: 6 February 2013

Published: 12 February 2013

\section{References}

1. Benedek GB, Villars FMH: Physics: With Illustrative Examples From Medicine and Biology: Electricity and Magnetism. New York: Springer; 2000. p. 403.

2. Alberts B, Bray D, Lewis J, Raff M, Roberts MK, Watson JD: Molecular Biology of the Cell. New York: Garland Publishing Company; 1994. p. 920

3. Gagliardi LJ: Electrostatic force generation in chromosome motions during mitosis. J Electrostat 2005 63:309-327.

4. Amirand $\mathrm{C}$, et al: Intracellular pH in one-cell mouse embryo differs between sub-cellular compartments and between interphase and mitosis. Biol Cell 2000, 92:409-419.

5. Steinhardt RA, Morisawa M: In Intracellular pH: Its Measurement, Regulation, and Utilization in Cellular Functions. Edited by Nuccitelli R, Deamer DW, Liss AR. New York: 1982:361-374.

6. Schatten G, Bestor T, Balczon R, Henson J, Schatten H: Intracellular pH shift leads to microtubule assembly and microtubule-mediated motility during sea urchin fertilization: correlations between elevated intracellular $\mathrm{pH}$ and microtubule activity and depressed intracellular pH and microtubule disassembly. Eur J Cell Biol 1985, 36:116-127.

7. Kirschner MW: Implications of treadmilling for the stability and polarity of actin and tubulin polymers in vivo. J Cell Biol 1980, 86:330-334

8. Deery WJ, Brinkley BR: Cytoplasmic microtubule assembly - disassembly from endogenous tubulin in a Brij lysed cell model. J Cell Biol 1983, 96:1631-1641.

9. Olmsted JB, Borisy GG: Characterization of microtubule assembly in porcine brain extracts by viscometry. Biochem. 1973, 12:4282-4289.

10. Jordan-Lloyd D, Shore A: The Chemistry of Proteins. J. A: Churchill Publishing Company; 1938

11. Pauling L: The adsorption of water by proteins. J Am Chem Soc 1945, 67:555-557.

12. Toney MF, Howard JN, Richer J, Borges GL, Gordon JG, Melroy OR, Wiesler DG, Yee D, Sorensen L: Voltagedependent ordering of water molecules at an electrode-electrolyte interface. Nature 1994, 368:444-446.

13. Teschke O, Ceotto G, De Souza EF: Interfacial water dielectric permittivity profile measurements using atomic force spectroscopy. Physical Review E 2001, 64. 011605-1 - 011605-10.

14. Gagliardi LJ: Induced electrostatic charge in poleward motion of chromosomes during mitosis. J Electrostat 2008, 66:147-155.

15. Gagliardi LJ: Electrostatic Considerations in Mitosis. Bloomington, IN: iUniverse Publishing Company; 2009.

16. Satarić MV, Tuszyński JA, Zakula RB: Kinklike excitations as an energy-transfer mechanism in microtubules. Physical Review E 1993, 48:589-597.

17. Brown JA, Tuszyński JA: Dipole interactions in axonal microtubules as a mechanism of signal propagation. Physical Review E 1997, 56:5834-5340.

18. Baker NA, Sept D, Joseph S, Holst MJ, McCammon JA: Electrostatics of nanosystems: applications to microtubules and the ribosome. Proc Natl Acad Sci 2001, 98:10037-10041.

19. Tuszyński JA, Brown JA, Hawrylak P: Dielectric polarization, electrical conduc-tion, information processing and quantum computation in microtubules. Are they plausible? Phil Trans R Soc (London) 1998, A356:1897-1926. 
20. Tuszyński JA, Brown JA, Carpenter EJ, Crawford E: Proceedings of the Electrostatics Society of America and Institute of Electrostatics - Japan. In ESA-IEJ Joint Symposium on Electrostatics. Edited by Crowley JM. CA: Laplacian Press; 2002:41-50.

21. Tuszyński JA, Hameroff S, Satarić MV, Trpisová B, Nip MLA: Ferroelectric behavior in microtubule dipole lattices: implications for information processing, signaling, and assembly/disassembly. J Theoretical Biol 1995, 174:371-380.

22. Stracke R, Böhm KJ, Wollweber L, Tuszyński JA, Unger E: Analysis of the migration behavior of single microtubules in electric fields. Biochem Biophys Res Commun 2002, 293:602-609.

23. Sackett D: pH-induced conformational changes in the carboxy-terminal tails of tubulin, 'Molecular Biophysics of the Cytoskeleton, Alberta, Canada: Banff; 1997.

24. Gagliardi LJ: Electrostatic force in prometaphase, metaphase, and anaphase-A chromosome motions. Physical Review E 2002, 66. 011901-1 - 011901-8.

25. Weisenberg RC: Microtubule formation in vitro in solutions containing low calcium concentrations. Science 1972, 177:1104-1105.

26. Borisy GG, Olmsted JB: Nucleated assembly of microtubules in porcine brain extracts. Science 1972 177:1196-1197.

27. Gagliardi LJ: Microscale electrostatics in mitosis. J Electrostat. 2002, 54:219-232.

28. Maiato H, DeLuca J, Salmon ED, Earnshaw WC: The dynamic kinetochore microtubule interface. J Cell Sci 2004, 117:5461-5477.

29. Hepler PK, Callaham DA: Free calcium increases in anaphase in stamen hair cells of Trandescantia. J Cell Biol 1987, 105:2137-2143.

30. Zhang DH, Callaham DA, Hepler PK: Regulation of anaphase chromosome motion in Tradescantia stamen hair cells by calcium and related signaling agents. J Cell Biol 1990, 111:171-182.

doi:10.1186/1742-4682-10-8

Cite this article as: Gagliardi and Shain: Is intracellular pH a clock for mitosis?. Theoretical Biology and Medical Modelling 2013 10:8.

\section{Submit your next manuscript to BioMed Central and take full advantage of:}

- Convenient online submission

- Thorough peer review

- No space constraints or color figure charges

- Immediate publication on acceptance

- Inclusion in PubMed, CAS, Scopus and Google Scholar

- Research which is freely available for redistribution

Submit your manuscript at www.biomedcentral.com/submit 